\title{
14 New Methods to Prove the Pythagorean Theorem by using Similar Triangles
}

\author{
Salman Mahmud* \\ Student of BIAM Model School and College, Bogura Bangladesh.
}

*Corresponding Author: Salman Mahmud, Student of BIAM Model School and College, Bogura Bangladesh.

\begin{abstract}
There seems to be about 500 different proofs of the Pythagorean theorem. Here in this paper I will show 14 new methods of proving the theorem by using similar triangles. Firstly, I will divide the a right triangle into two new right triangles and then there will be three similar right triangles. Secondly, basing on the proportionality of these similar triangles, I will get 22 equations. Comparing the equations I will demonstrate the 14 new methods one by one.
\end{abstract}

Keywords: Pythagorean theorem, right angled triangle, Pythagoras, similar triangles, new proofs, new methods.

\section{INTRODUCTION}

There is an abundance of proofs available for Pythagoras' Theorem on right-angled triangles, from Pythagoras' own alleged proof in the 6th century B.C., through Euclid's proof, the proof by Thabit ibn Qurra of Baghdad in the $9^{\text {th }}$ century, the Indian 12th century mathematician Bhaskara's proof, to the one by the 20th President of the United States James Garfield, who published his paper in 1876, five years before taking up office as President.

The main aim of this paper is to demonstrate 14 different methods for proving Pythagorean theorem. A theorem can be proved by so many different ways. But if someone asks, "is there a theorem in Euclidean geometry that has the most number of proofs?" The answer is yes and the theorem is the famous Pythagorean theorem. It is believed that Pythagoras lived in the 6th century B. C. in the island of Samos, Egypt and in Croton in southern Italy, besides visits to neighbouring countries. He was the leader of a society, called Brotherhood, which was devoted to the study of mathematics, astronomy, religion and music. Among other things he is famous for the theorem attributed to him. The theorem was known earlier in some form or other in India and China; the Hindu mathematician Baudhayana discussed it around 800 B.C. in his book Baudhayana Sulba Sutra. It was known even earlier to the Babylonians. Pythagoras seems to have been the one who formulated it in a form such that he is considered as the first pure mathematician in history. The theorem is called Pythagorean sometimes due to the secretive nature of his society. Pythagorean Theorem becomes an important base in the calculation of the length side of the flat straight sides with the help of right-angled triangles, because the Pythagorean Theorem is a fundamental theorem in mathematics. The Pythagorean Theorem has been introduced to students from elementary school until secondary school. Pythagoras discovery in the field of music and mathematics remains alive today. Pythagorean Theorem is taught in schools and used to calculate the distance a side of a right triangle. Before Pythagoras, there were no proofs or assumptions underlying on right triangle systematic.

The famous Pythagorean Theorem states that the square of the hypotenuse of a right triangle is equal to the sum of each other sides square. Although the development of the various version of other proofs has been widely known before Pythagoras, all proof related to the right triangle is still addressed to the Pythagorean Theorem, because he was the first to prove the observation mathematically in a right triangle. One of the benefits of this theorem is as a tool in the calculation of the natural phenomena. The Pythagorean Theorem was a base of proving Fermat's theorem in 1620: $\mathrm{xn}+\mathrm{yn}=\mathrm{zn}$, which was firstly proven by Sir Andrew Wiles in 1994.After that, some math calculations in a quite complicated technique were resolved. 
This study will help us to know more different uses of similar triangles in case of proving Pythagorean theorem. The students as well as the teachers will be able to understand the theorem easily.

\section{DISCUSSION AND RESULTS}

The theorem that we are setting out to prove, the Pythagorean Theorem, says the following. Take any right-angled triangle, in which the hypotenuse has a length of $c$ and the other two sides lengths of a and $b$. The theorem asserts that $a^{2}+b^{2}=c^{2}$.

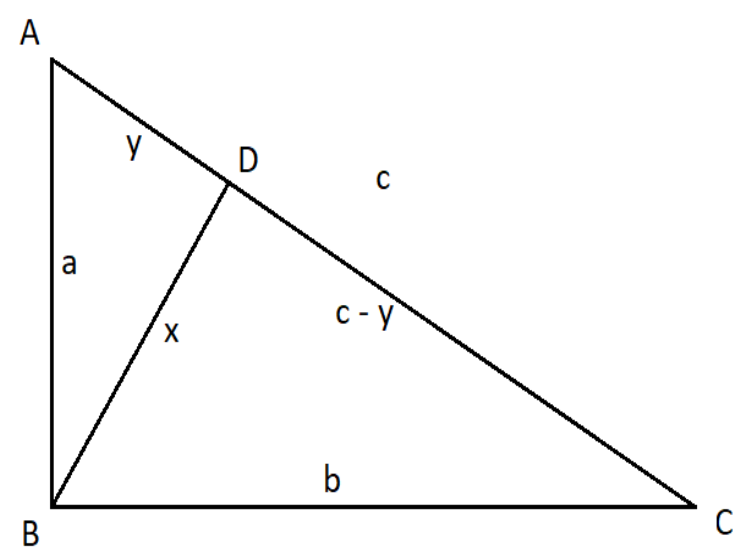

Figure1. Here $A B C$ is a right-angled triangle and $B D \perp A C$.

Here, $\mathrm{AB}=\mathrm{a}, \mathrm{BC}=\mathrm{b}, \mathrm{AC}=\mathrm{c}, \mathrm{BD}=\mathrm{x}, \mathrm{AD}=\mathrm{y}$ that's why $\mathrm{CD}=\mathrm{c}-\mathrm{y} . \triangle \mathrm{ABC}, \triangle \mathrm{ABD}$ and $\triangle \mathrm{BCD}$ are right triangles and they are similar.

Now $\triangle \mathrm{BCD}$ and $\triangle \mathrm{ABD}$ are similar. That's why,

$$
\begin{aligned}
& \frac{x}{y}=\frac{c-y}{x} \\
& \Rightarrow x^{2}=c y-y^{2} \\
& x=\sqrt{c y-y^{2}}
\end{aligned}
$$

Again,

$\frac{\mathrm{x}}{\mathrm{y}}=\frac{\mathrm{b}}{\mathrm{a}}$

$\Rightarrow \mathrm{x}=\frac{\mathrm{by}}{\mathrm{a}}$

Again, $\quad \frac{c-y}{x}=\frac{b}{a}$

$\Rightarrow \mathrm{x}=\frac{\mathrm{a}(\mathrm{c}-\mathrm{y})}{\mathrm{b}}$

$\triangle \mathrm{ABD}$ and $\triangle \mathrm{ABC}$ are similar. That's why,

$\frac{\mathrm{x}}{\mathrm{b}}=\frac{\mathrm{y}}{\mathrm{a}}$

$\Rightarrow \mathrm{x}=\frac{\mathrm{by}}{\mathrm{a}}$

Again, $\quad \frac{\mathrm{x}}{\mathrm{b}}=\frac{\mathrm{a}}{\mathrm{c}}$

$\Rightarrow \mathrm{x}=\frac{\mathrm{ab}}{\mathrm{c}}$

Again, $\quad \frac{\mathrm{y}}{\mathrm{a}}=\frac{\mathrm{a}}{\mathrm{c}}$

$\Rightarrow \mathrm{a}^{2}=\mathrm{yc}$

$\triangle \mathrm{BCD}$ and $\triangle \mathrm{ABC}$ are similar. That's why, $\frac{\mathrm{x}}{\mathrm{a}}=\frac{\mathrm{c}-\mathrm{y}}{\mathrm{b}}$ 
$\Rightarrow \mathrm{x}=\frac{\mathrm{a}(\mathrm{c}-\mathrm{y})}{\mathrm{b}}$

Again, $\quad \frac{\mathrm{x}}{\mathrm{a}}=\frac{\mathrm{b}}{\mathrm{c}}$

$\Rightarrow \mathrm{x}=\frac{\mathrm{ab}}{\mathrm{c}}$

Again, $\quad \frac{\mathrm{c}-\mathrm{y}}{\mathrm{b}}=\frac{\mathrm{b}}{\mathrm{c}}$

$\Rightarrow \mathrm{b}^{2}=\mathrm{c}^{2}-\mathrm{cy}$

Combining Eq.[2] or Eq.[4] and Eq.[3] or Eq.[7] we get,

$$
\begin{aligned}
& \frac{b y}{a}=\frac{a(c-y)}{b} \\
& \Rightarrow b^{2} y=a^{2} c-a^{2} y \\
& \Rightarrow b^{2} y+a^{2} y=a^{2} c \\
& \Rightarrow y\left(a^{2}+b^{2}\right)=a^{2} c \\
& y=\frac{a^{2} c}{a^{2}+b^{2}}
\end{aligned}
$$

Combining Eq.[2] or Eq.[4] and Eq.[5] or Eq.[8] we get,

$\frac{\mathrm{by}}{\mathrm{a}}=\frac{\mathrm{ab}}{\mathrm{c}}$

$\Rightarrow \mathrm{bcy}=\mathrm{a}^{2} \mathrm{~b}$

$\mathrm{y}=\frac{\mathrm{a}^{2}}{\mathrm{c}}$

Combining Eq.[1] and Eq.[2] or Eq.[4] we get,

$$
\begin{aligned}
& \sqrt{c y-y^{2}}=\frac{b y}{a} \\
& \Rightarrow c y-y^{2}=\frac{b^{2} y^{2}}{a^{2}} \\
& \Rightarrow a^{2} c y-a^{2} y^{2}=b^{2} y^{2} \\
& \Rightarrow y\left(a^{2} c-a^{2} y\right)=b^{2} y^{2} \\
& \Rightarrow a^{2} y+b^{2} y^{2}=a^{2} c \\
& y=\frac{a^{2} c}{a^{2}+b^{2}}
\end{aligned}
$$

Combining Eq.[3] or Eq.[7] and Eq.[5] or Eq.[8] we get,

$$
\begin{aligned}
& \frac{a(c-y)}{b}=\frac{a b}{c} \\
& \Rightarrow a c^{2}-a c y=a b^{2} \\
& \Rightarrow c^{2}-c y=b^{2} \\
& y=\frac{c^{2}-b^{2}}{c}
\end{aligned}
$$

Combining Eq.[1] and Eq.[10] or Eq.[12] we get,

$$
\begin{aligned}
& x=\sqrt{c \times \frac{a^{2} c}{a^{2}+b^{2}}-\left(\frac{a^{2} c}{a^{2}+b^{2}}\right)^{2}} \\
& \Rightarrow x=\sqrt{\frac{a^{4} c^{2}+a^{2} b^{2} c^{2}-a^{4} c^{2}}{\left(a^{2}+b^{2}\right)^{2}}} \\
& x=\frac{a b c}{a^{2}+b^{2}}
\end{aligned}
$$


Combining Eq.[1] and Eq.[11] we get,

$\mathrm{x}=\sqrt{\mathrm{c} \times \frac{\mathrm{a}^{2}}{\mathrm{c}}-\left(\frac{\mathrm{a}^{2}}{\mathrm{c}}\right)^{2}}$

$\Rightarrow \mathrm{x}=\sqrt{\mathrm{a}^{2}-\frac{\mathrm{a}^{4}}{\mathrm{c}^{2}}}$

$\mathrm{x}=\frac{\sqrt{\mathrm{a}^{2} \mathrm{c}^{2}-\mathrm{a}^{4}}}{\mathrm{c}}$

Combining Eq.[1] and Eq.[13] we get,

$$
\begin{aligned}
& x=\sqrt{c \times \frac{c^{2}-b^{2}}{c}-\left(\frac{c^{2}-b^{2}}{c}\right)^{2}} \\
& \Rightarrow x=\sqrt{\frac{c^{4}-c^{2} b^{2}-c^{4}+2 c^{2} b^{2}-b^{4}}{c^{2}}} \\
& x=\frac{\sqrt{b^{2} c^{2}-b^{4}}}{c}
\end{aligned}
$$

Combining Eq.[2] or Eq.[4] and Eq.[10] or Eq.[12] we get,

$$
\begin{aligned}
& x=\frac{b \times \frac{a^{2} c}{a^{2}+b^{2}}}{a} \\
& \Rightarrow x=\frac{a b c}{a^{2}+b^{2}}
\end{aligned}
$$

Combining Eq.[2] or [4] and Eq.[11] we get,

$x=\frac{b \times \frac{a^{2}}{c}}{a}$
$\Rightarrow x=\frac{a b}{c}$

Combining Eq.[2] or Eq.[4] and Eq.[13] we get,

$\mathrm{x}=\frac{\mathrm{b}\left(\mathrm{c}^{2}-\mathrm{b}^{2}\right)}{\mathrm{ac}}$

Combining Eq.[3] or Eq.[7] and Eq.[10] or Eq.[12] we get,

$$
\begin{aligned}
& x=\frac{a\left(c-\frac{a^{2} c}{a^{2}+b^{2}}\right)}{b} \\
& \Rightarrow x=\frac{a\left(\frac{a^{2} c+b^{2} c-a^{2} c}{a^{2}+b^{2}}\right)}{b} \\
& x=\frac{a b c}{a^{2}+b^{2}}
\end{aligned}
$$

Combining Eq.[3] or Eq.[7] and Eq.[11] we get,

$$
\begin{aligned}
& x=\frac{a\left(c-\frac{a^{2}}{c}\right)}{b} \\
& \Rightarrow x=\frac{a\left(c^{2}-a^{2}\right)}{b c}
\end{aligned}
$$

Combining Eq.[3] or Eq.[7] and Eq.[13] we get,

$$
\begin{aligned}
& x=\frac{a\left(c-\frac{c^{2}-b^{2}}{c}\right)}{b} \\
& \Rightarrow x=\frac{a\left(\frac{c^{2}-c^{2}+b^{2}}{c}\right)}{b} \\
& \Rightarrow x=\frac{a b}{c}
\end{aligned}
$$


Combining Eq.[10] or Eq.[12] and Eq.[11] we get,

$\frac{\mathrm{a}^{2} \mathrm{c}}{\mathrm{a}^{2}+\mathrm{b}^{2}}=\frac{\mathrm{a}^{2}}{\mathrm{c}}$

$\Rightarrow a^{2} c^{2}=a^{4}+a^{2} b^{2}$

$\Rightarrow \mathrm{a}^{2}\left(\mathrm{a}^{2}+\mathrm{b}^{2}\right)=\mathrm{a}^{2} \mathrm{c}^{2}$

$a^{2}+b^{2}=c^{2} \quad$ [Method no. 1]

Combining Eq.[10] or Eq.[12] and Eq.[13] we get,

$\frac{\mathrm{a}^{2} \mathrm{c}}{\mathrm{a}^{2}+\mathrm{b}^{2}}=\frac{\mathrm{c}^{2}-\mathrm{b}^{2}}{\mathrm{c}}$

$\Rightarrow a^{2} c^{2}=a^{2} c^{2}-a^{2} b^{2}+b^{2} c^{2}-b^{4}$

$\Rightarrow a^{2} c^{2}-a^{2} c^{2}+a^{2} b^{2}+b^{4}=b^{2} c^{2}$

$\Rightarrow b^{2}\left(a^{2}+b^{2}\right)=b^{2} c^{2}$

$a^{2}+b^{2}=c^{2}$ [Method no. 2]

Combining Eq.[11] and Eq.[13] we get,

$\frac{\mathrm{a}^{2}}{\mathrm{c}}=\frac{\mathrm{c}^{2}-\mathrm{b}^{2}}{\mathrm{c}}$

$\Rightarrow \mathrm{a}^{2}=\mathrm{c}^{2}-\mathrm{b}^{2}$

$\mathrm{a}^{2}+\mathrm{b}^{2}=\mathrm{c}^{2}$ [Method no. 3]

Combining Eq.[15] and Eq.[16] we get,

$\frac{\sqrt{a^{2} c^{2}-a^{4}}}{c}=\frac{\sqrt{b^{2} c^{2}-b^{4}}}{c}$

$\Rightarrow \mathrm{a}^{2} \mathrm{c}^{2}-\mathrm{a}^{4}=\mathrm{b}^{2} \mathrm{c}^{2}-\mathrm{b}^{4}$

$\Rightarrow \mathrm{a}^{2} \mathrm{c}^{2}-\mathrm{b}^{2} \mathrm{c}^{2}=\mathrm{a}^{4}-\mathrm{b}^{4}$

$\Rightarrow \mathrm{c}^{2}\left(\mathrm{a}^{2}-\mathrm{b}^{2}\right)=\left(\mathrm{a}^{2}+\mathrm{b}^{2}\right)\left(\mathrm{a}^{2}-\mathrm{b}^{2}\right)$

$a^{2}+b^{2}=c^{2} \quad$ [Method no. 4]

Combining Eq.[14] or Eq.[17] or Eq.[20] and Eq. [18] or Eq.[22] we get,

$\frac{\mathrm{abc}}{\mathrm{a}^{2}+\mathrm{b}^{2}}=\frac{\mathrm{ab}}{\mathrm{c}}$

$\Rightarrow \mathrm{ab}\left(\mathrm{a}^{2}+\mathrm{b}^{2}\right)=\mathrm{abc}^{2}$

$a^{2}+b^{2}=c^{2} \quad[$ Method no. 5]

Combining Eq.[14] or Eq.[17] or Eq.[20] and Eq.[21] we get,

$\frac{a b c}{a^{2}+b^{2}}=\frac{a\left(c^{2}-a^{2}\right)}{b c}$

$\Rightarrow b^{2} c^{2}=a^{2} c^{2}-a^{4}+b^{2} c^{2}-a^{2} b^{2}$

$\Rightarrow b^{2} c^{2}-b^{2} c^{2}+a^{4}+a^{2} b^{2}=a^{2} c^{2}$

$\Rightarrow \mathrm{a}^{2}\left(\mathrm{a}^{2}+\mathrm{b}^{2}\right)=\mathrm{a}^{2} \mathrm{c}^{2}$

$a^{2}+b^{2}=c^{2} \quad[$ Method no. 6]

Combining Eq. [14] or Eq.[17] or Eq.[20] and Eq.[19] we get,

$$
\begin{aligned}
& \frac{a b c}{a^{2}+b^{2}}=\frac{b\left(c^{2}-b^{2}\right)}{a c} \\
& \Rightarrow a^{2} c^{2}=a^{2} c^{2}-a^{2} b^{2}+b^{2} c^{2}-b^{4} \\
& \Rightarrow b^{2}\left(a^{2}+b^{2}\right)=b^{2} c^{2}
\end{aligned}
$$


$\mathrm{a}^{2}+\mathrm{b}^{2}=\mathrm{c}^{2} \quad$ [Method no. 7]

Combining Eq.[15] and Eq.[18] or Eq.[22] we get,

$\frac{\sqrt{a^{2} c^{2}-a^{4}}}{c}=\frac{a b}{c}$

$\Rightarrow \mathrm{a}^{2} \mathrm{c}^{2}-\mathrm{a}^{4}=\mathrm{a}^{2} \mathrm{~b}^{2}$

$\Rightarrow \mathrm{a}^{4}+\mathrm{a}^{2} \mathrm{~b}^{2}=\mathrm{a}^{2} \mathrm{c}^{2}$

$\Rightarrow \mathrm{a}^{2}\left(\mathrm{a}^{2}+\mathrm{b}^{2}\right)=\mathrm{a}^{2} \mathrm{c}^{2}$

$\mathrm{a}^{2}+\mathrm{b}^{2}=\mathrm{c}^{2} \quad$ [Method no. 8]

Combining Eq.[16] and Eq.[18] or Eq.[22] we get,

$\frac{\sqrt{\mathrm{b}^{2} \mathrm{c}^{2}-\mathrm{b}^{4}}}{\mathrm{c}}=\frac{\mathrm{ab}}{\mathrm{c}}$

$\Rightarrow \mathrm{b}^{2} \mathrm{c}^{2}-\mathrm{b}^{4}=\mathrm{a}^{2} \mathrm{~b}^{2}$

$\Rightarrow \mathrm{a}^{2} \mathrm{~b}^{2}+\mathrm{b}^{4}=\mathrm{b}^{2} \mathrm{c}^{2}$

$\Rightarrow \mathrm{b}^{2}\left(\mathrm{a}^{2}+\mathrm{b}^{2}\right)=\mathrm{b}^{2} \mathrm{c}^{2}$

$a^{2}+b^{2}=c^{2} \quad$ [Method no. 9]

Combining Eq.[19] and Eq.[18] or Eq.[22] we get,

$\frac{\mathrm{b}\left(\mathrm{c}^{2}-\mathrm{b}^{2}\right)}{\mathrm{ac}}=\frac{\mathrm{ab}}{\mathrm{c}}$

$\Rightarrow \mathrm{b}\left(\mathrm{c}^{2}-\mathrm{b}^{2}\right)=\mathrm{a}^{2} \mathrm{~b}$

$\Rightarrow \mathrm{c}^{2}-\mathrm{b}^{2}=\mathrm{a}^{2}$

$a^{2}+b^{2}=c^{2} \quad$ [Method no. 10]

Combining Eq.[21] and Eq.[18] or Eq.[22] we get,

$\frac{\mathrm{a}\left(\mathrm{c}^{2}-\mathrm{a}^{2}\right)}{\mathrm{bc}}=\frac{\mathrm{ab}}{\mathrm{c}}$

$\Rightarrow \mathrm{a}\left(\mathrm{c}^{2}-\mathrm{a}^{2}\right)=\mathrm{ab}^{2}$

$\Rightarrow \mathrm{c}^{2}-\mathrm{a}^{2}=\mathrm{b}^{2}$

$\mathrm{a}^{2}+\mathrm{b}^{2}=\mathrm{c}^{2} \quad$ [Method no. 11]

Combining Eq.[19] and Eq.[21] we get,

$\frac{\mathrm{b}\left(\mathrm{c}^{2}-\mathrm{b}^{2}\right)}{\mathrm{ac}}=\frac{\mathrm{a}\left(\mathrm{c}^{2}-\mathrm{a}^{2}\right)}{\mathrm{bc}}$

$\Rightarrow \mathrm{b}^{2}\left(\mathrm{c}^{2}-\mathrm{b}^{2}\right)=\mathrm{a}^{2}\left(\mathrm{c}^{2}-\mathrm{a}^{2}\right)$

$\Rightarrow b^{2} c^{2}-b^{4}=a^{2} c^{2}-a^{4}$

$\Rightarrow b^{2} c^{2}-a^{2} c^{2}=b^{4}-a^{4}$

$\Rightarrow c^{2}\left(b^{2}-a^{2}\right)=\left(b^{2}+a^{2}\right)\left(b^{2}-a^{2}\right)$

$a^{2}+b^{2}=c^{2} \quad$ [Method no. 12]

Combining Eq.[16] and Eq.[19] we get,

$$
\begin{aligned}
& \frac{\sqrt{b^{2} c^{2}-b^{4}}}{c}=\frac{b\left(c^{2}-b^{2}\right)}{a c} \\
& \Rightarrow a^{2}\left(b^{2} c^{2}-b^{4}\right)=b^{2}\left(c^{2}-b^{2}\right)^{2} \\
& \Rightarrow a^{2} b^{2}\left(c^{2}-b^{2}\right)=b^{2}\left(c^{2}-b^{2}\right)^{2} \\
& \Rightarrow a^{2}=c^{2}-b^{2}
\end{aligned}
$$


$a^{2}+b^{2}=c^{2} \quad[$ Method no. 13]

Combining Eq.[15] and Eq.[21] we get,

$\frac{\sqrt{a^{2} c^{2}-a^{4}}}{c}=\frac{a\left(c^{2}-a^{2}\right)}{b c}$

$\Rightarrow \mathrm{b} \sqrt{\mathrm{a}^{2}\left(\mathrm{c}^{2}-\mathrm{a}^{2}\right)}=\mathrm{a}\left(\mathrm{c}^{2}-\mathrm{a}^{2}\right)$

$\Rightarrow \mathrm{ab} \sqrt{\left(\mathrm{c}^{2}-\mathrm{a}^{2}\right)}=\mathrm{a}\left(\mathrm{c}^{2}-\mathrm{a}^{2}\right)$

$\Rightarrow \mathrm{b}^{2}\left(\mathrm{c}^{2}-\mathrm{a}^{2}\right)=\left(\mathrm{c}^{2}-\mathrm{a}^{2}\right)^{2}$

$\Rightarrow \mathrm{b}^{2}=\mathrm{c}^{2}-\mathrm{a}^{2}$

$a^{2}+b^{2}=c^{2} \quad[$ Method no. 14]

\section{CONCLUSION}

The above discussion has discovered the fourteen new ways of proving Pythagorean Theorem. Using these methods we can easily prove the Pythagorean theorem by using only the proportionality of similar triangles. This study also shows us all the methods of proving the Pythagorean theorem by using only one well-known figure [1]. However, the fourteen new evidences above are pretty easy to be understood by teachers and students in the school.

\section{ACKNOWLEDGEMENT}

I should thank MD. Shah Alam and MST. Sabina Yesmin for providing me with the help materials.

\section{AUTHOR'S BIOGRAPHY}

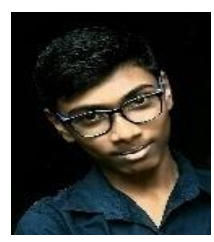

Salman Mahmud, is s an undergraduate student of BIAM Model school and College, Bogura, Bangladesh. His core research interest is in modern physics. But he is interested in all the sectors of science.

[1] S. Mahmud, (2019) A New Long Proof of the Pythagorean Theorem, International Journal of Scientific and Innovative Mathematical Research vol. 7, no. 9, pp. 3-7, 2019. DOI: http://dx.org/10.20431/23473142.0709002

[2] Swaminathan S (2014). The Pythagorean Theorem, Journal of Biodiversity, Bioprospecting and Development. vol.1, issue 3, doi :10.4172/2376-0214.1000128

[3] B. Kaushik, (2015). "A New and Very Long Proof of the Pythagoras Theorem By Way of a Proposition on Isosceles Triangles," MPRA Paper 61125, University Library of Munich, Germany. https://ideas.repec.org/p/pra/mprapa/61125.html

[4] S. Mahmud, (2019). Calculating the area of the Trapezium by Using the Length of the Non Parallel Sides: A New Formula for Calculating the area of Trapezium. International Journal of Scientific and Innovative Mathematical Research, volume 7, issue 4, pp.25-27.http://dx.doi.org/10.20431/2347-3142.0704004

[5] L. Nurul , H., D., (2017). Five New Ways to Prove a Pythagorean Theorem, International Journal of Advanced Engineering Research and Science, volume 4, issue 7, pp.132-137 http://dx.doi.org/ 10.22161/ijaers.4.7.21

Citation: Salman Mahmud, 14 New Methods to Prove the Pythagorean Theorem by using Similar Triangles, International Journal of Scientific and Innovative Mathematical Research (IJSIMR), vol. 8, no. 2, pp. 22-28, 2020. Available : DOI: http://dx.doi.org/10.20431/2347-3142.0802003

Copyright: (c) 2020 Authors. This is an open-access article distributed under the terms of the Creative Commons Attribution License, which permits unrestricted use, distribution, and reproduction in any medium, provided the original author and source are credited. 